\title{
Pressure-induced phase transitions in coesite
}

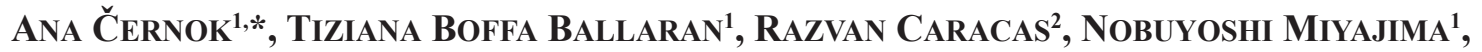

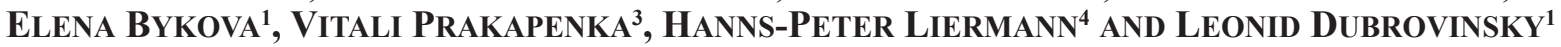

\author{
${ }^{1}$ Bayerisches Geoinstitut, Universität Bayreuth, Universitätsstrasse 30, D-95440 Bayreuth, Germany \\ ${ }^{2}$ Centre National de la Recherche Scientifique Laboratoire de Geologie de Lyon (LGLTPE) UMR 5276 Ecole Normale Supérieure de Lyon 46, \\ allée d'Italie, 69364 Lyon, France \\ ${ }^{3}$ Center for Advanced Radiation Sources, University of Chicago, Argonne National Laboratory, Building 434A, 9700 South Cass Avenue, \\ Argonne, Illinois 60439, U.S.A. \\ ${ }^{4}$ Photon Sciences, Deutsches Elektronen-Synchrotron (DESY), Notkestrasse 85, 22607 Hamburg, Germany
}

\begin{abstract}
High-pressure behavior of coesite was studied on single crystals using diamond-anvil cells with neon as the pressure-transmitting medium by means of in situ Raman spectroscopy up to pressures of $\sim 51 \mathrm{GPa}$. The experimental observations were complemented with theoretical computations of the Raman spectra under similar pressure conditions. We find that coesite undergoes two phase transitions and does not become amorphous at least up to $\sim 51 \mathrm{GPa}$. The first phase transition (coesite I to coesite II) is reversible and occurs around $23 \mathrm{GPa}$. The second transition (coesite II to coesite III) at about 35 $\mathrm{GPa}$ is also reversible but involves a large hysteresis. Samples recovered from the highest pressure achieved, $51 \mathrm{GPa}$, show Raman spectra of the initial coesite. The ab initio calculations gave insight into the initiation mechanism of the first phase transition, implying, from the analysis of unstable phonon modes, that it is probably a displacive phase transition due to shearing of the four-membered rings of $\mathrm{SiO}_{4}$ tetrahedra upon compression. The transition to the lowest-symmetry phase, coesite III, is possibly a first-order phase transition that leads to a very distinct structure. None of the metastable high-pressure phases of coesite has been previously studied and it was widely accepted that coesite undergoes pressure-induced amorphization at significantly lower pressures $(30 \mathrm{GPa})$. The study of the high-pressure behavior of coesite is important to better constrain the metastable phase diagram of silica. Further crystallographic investigations are necessary for characterizing the structures of these metastable coesite forms. Crystalline or amorphous metastable phases derived from coesite under high-pressure conditions are of particular interest because they can be used as potential tracers of peak transient pressures (stress) reached in processes such as impacts or faulting.

Keywords: Silica, coesite, high-pressure polymorph, metastable, diamond-anvil cell, high-pressure
\end{abstract} Raman spectra

\section{INTRODUCTION}

Silica, $\mathrm{SiO}_{2}$, exhibits a very rich polymorphism with more than 30 stable or metastable phases, most of which occur at ambient to moderate pressures ( $<9 \mathrm{GPa}$ ). Some of these phases, such as quartz, trydimite, and cristobalite, consist of frameworks of $\mathrm{SiO}_{4}$ tetrahedra and are abundant in nature. Phases of silica stable at higher pressures and temperatures are mostly composed of octahedrally coordinated silicon atoms. Coesite, thermodynamically stable above ca. $2.5 \mathrm{GPa}$ and at temperatures in excess of $500^{\circ} \mathrm{C}$, is the densest known polymorph with silicon atom tetrahedrally coordinated to oxygen. This polymorph was first observed experimentally by Coes in 1953 and later discovered in nature by Chao and coworkers, in impact breccia from Meteor Crater (Chao et al. 1960). Nowadays coesite is widely accepted as a high-pressure indicator in rocks related to meteorite impact sites, where it forms due to the high-pressure and high-temperature regime of the impact

\footnotetext{
* E-mail: Ana.Cernok@uni-bayreuth.de
}

and to the very rapid quenching conditions that prevent a reversion from coesite to quartz (e.g., Xiao et al. 2011). Shock-induced formation of coesite has been also observed in meteorites, where it can be found along with other shock minerals (e.g., Ohtani et al. 2011). Terrestrial occurrence of coesite was reported from deeply $(>100 \mathrm{~km}$ ) subducted crustal rocks (ultrahigh-pressure metamorphic rocks) or mantle derived rocks (kimberlites), which have been exhumed under conditions that prevent retrograde transition to quartz (e.g., Smyth and Hatton 1977; Chopin 1984; Mosenfelder and Bohlen 1997; Parkinson 2000; Korsakov et al. 2007; Ruiz-Cruz and Sanz de Galdeano 2012). In these rocks, coesite commonly occurs as an inclusion within clinopyroxene, olivine, garnet, or diamond. Coesite-in-diamond assemblage was recently introduced as an important high-pressure barometer (Sobolev et al. 2000).

Because of the relatively strong Si-O bonding in silica, there are high kinetic barriers associated with the transitions to stable high-pressure phases consisting of $\mathrm{SiO}_{6}$ octahedra (Haines et al. 2001). This results in complicated metastable phenomena at high 
pressures and ambient temperature such as the persistence of low-pressure phases far out of their stability fields and transitions to metastable crystalline and amorphous phases. Slow kinetics, enhanced metastability, and formation of poorly crystallized or structurally disordered materials usually give rise to weak X-ray diffraction patterns that are difficult to interpret, resulting in contradictory interpretations. Another major complexity in studying the behavior of silica also arises from the fact that the transition mechanisms, the pathways through intermediate metastable phases and the onset of pressure-induced amorphization strongly depend on the starting polymorph, as well as on the hydrostaticity and stress anisotropy present in the sample during experiments. For example, experimental studies on polycrystalline $\alpha$-quartz in neon pressure medium (Kingma et al. 1993) evidenced pressure-induced phase transitions above $21 \mathrm{GPa}$ to a crystalline metastable phase of reduced symmetry, followed by pressure-induced amorphization above $30 \mathrm{GPa}$. In helium pressure medium, which provides higher hydrostaticity, polycrystalline quartz is found to remain crystalline up to $45 \mathrm{GPa}$ and to transform to a monoclinic phase at such pressure (Haines et al. 2001). A transformation path through several metastable phases has been reported in cristobalite, as well (e.g., Yagi and Yamakata 2000; Prokopenko et al. 2001; Dera et al. 2011). Numerical calculations also stressed the fact that stable polymorphs of $\mathrm{SiO}_{2}$ may undergo several phase transitions to metastable phases with increasing pressure before reaching amorphization (Teter and Hemley 1998; Wentzcovitch et al. 1998; Dean et al. 2000).

In an experimental study on the high-pressure behavior of coesite, Hemley (1987) observed changes in the Raman spectra of coesite collected at ambient temperature and at the pressures of 22-25 GPa in argon pressure medium, and reported that coesite becomes amorphous above $30 \mathrm{GPa}$. The authors attributed such changes to a high-pressure phase transformation occurring in coesite; however, no further investigation has been reported to better describe such behavior.

In this study, we aim at giving insight into the high-pressure behavior of coesite. We measure in situ Raman spectra at pressures up to $51 \mathrm{GPa}$ and complement them with theoretical computations of Raman spectra under similar pressure conditions. We find two phase transitions clearly distinguishable by Raman spectroscopy and explain the mechanism of the first transition based on the theoretical calculations.

\section{EXPERIMENTAL METHODS}

\section{Synthesis}

The starting material for the coesite synthesis was $\mathrm{SiO}_{2}$ glass powder with very low trace elements content, as analyzed at the BGI using LA-ICP-MS: Al 20 ppm, Ge 1.3 ppm, Na 1.0 ppm, Li 0.8 ppm, and B, Ti, Fe, Ga, Rb, and Sn below the detection limits. Coesite single crystals were synthesized by mixing the starting powder with $\sim 5 \mathrm{wt} \%$ distilled water inside a platinum capsule, which was then welded shut. The capsule was first placed into pyrophyllite sleeves and then in a $0.5^{\prime \prime}$ talc-pyrex piston-cylinder assembly containing internal, tapered graphite resistance furnaces (Bromiley and Keppler 2004). The mixture was pressurized to $3.5 \mathrm{GPa}$ and slowly heated up to $1250{ }^{\circ} \mathrm{C}$, kept at this temperature for $\sim 15 \mathrm{~h}$, then cooled down to $1100{ }^{\circ} \mathrm{C}$ in $5 \mathrm{~h}$, and finally quenched. Slow cooling procedure and water-saturated conditions resulted in growth of relatively large (above 100 $\mu \mathrm{m}$ in linear dimensions) crystals. No Raman peaks were observed in the spectra of synthesized coesite in the O-H vibration region $\left(2800-3400 \mathrm{~cm}^{-1}\right)$. This is in agreement with the study on pressure dependence of hydrogen solubility in coesite (Mosenfelder 2000; Koch-Müller et al. 2001), according to which no water should be present in coesite synthesized at $3.5 \mathrm{GPa}$ and $1250{ }^{\circ} \mathrm{C}$.

\section{High-pressure Raman spectroscopy}

Piston-cylinder type diamond-anvil cells (DAC) developed at BGI (Kantor et al. 2012) made of high-temperature resistant alloy were used for the high-pressure experiments. Experiments in quasi-hydrostatic conditions were carried out using $250 \mu \mathrm{m}$ culet size diamonds, a cylindrical pressure chamber of $30-40 \mu \mathrm{m}$ height and $\sim 125 \mu \mathrm{m}$ diameter drilled in a pre-indented rhenium gasket. We used neon as pressure-transmitting medium, loaded using the BGI gas loading system (Kurnosov et al. 2008). Selected crystals were in the form of plates of typical size $15 \times 30 \times$ $50 \mu \mathrm{m}^{3}$ and they were loaded together with a $\sim 5 \mu \mathrm{m}$ in diameter ruby sphere for pressure determination. The pressure was determined using the ruby luminescence line (Mao et al. 1986).

Raman spectroscopy measurements were performed using a LabRam system (Horiba Scientific Inc.) with a He-Ne-laser (excitation wavelength $632.8 \mathrm{~nm}$, output power $0.15 \mathrm{~W}$, spectral resolution $2 \mathrm{~cm}^{-1}$ ) as well as Dilor XY Raman spectrometer with $\mathrm{Ar}+$ ion laser $(514.5 \mathrm{~nm}$, Coherent Innova 300, spectral resolution $1 \mathrm{~cm}^{-1}$ ). The output power was varied according to the sample signal between 0.3 and $1.0 \mathrm{~W}$ when using the Dilor instrument. The spectrometers were calibrated using either the silicon peak at $520 \mathrm{~cm}^{-1}$ or referencing the value of the absolute wavelength of the ruby $R_{1}$ fluorescence line. Raman spectra were collected between 200 and $1200 \mathrm{~cm}^{-1}$. This region is optimal because the LabRam spectrometer used in our experiments is equipped with a notch filter that prevents collection of the frequencies lower than $150 \mathrm{~cm}^{-1}$, whereas above $1200 \mathrm{~cm}^{-1}$ low-intensity peaks of coesite are suppressed by strong diamond peak centered near $1300 \mathrm{~cm}^{-1}$. We performed five different DAC experiments at room temperature by pressurizing the coesite crystals in steps of several GPa up to a maximum of $\sim 51 \mathrm{GPa}$. In two of these experiments, Raman spectra were also collected during decompression. Peak positions in the Raman spectra were determined by fitting to Lorentzian peak shape using Igor Pro v. 6.22 software.

\section{External electrical heating in DAC}

Heating experiments up to $\sim 400{ }^{\circ} \mathrm{C}$ were performed using platinum-wire resistive whole-cell heater. The heater has an external diameter of $50 \mathrm{~mm}$, it is made of fired pyrophyllite and $0.5 \mathrm{~mm}$ in diameter platinum wire is folded over the inner surface of the heater. For the purpose of protecting the Raman spectrometer from heating over several hours, we used a thermal insulator that is made of a square-shaped double-layered stainless steel box. Stainless steel of "type 1.4571" is oxidation-resistant at elevated temperatures and shows no oxidation effect to at least $600{ }^{\circ} \mathrm{C}$ (Fig. 1). The DAC and the whole-cell heater were fixed on a stainless steel holder, which was then placed inside the steel box with openings at the bottom and the top, to allow optical access. Temperature was measured using a Pt Pt/Rh S-type thermocouple placed in the vicinity of the gasket.

In the first experiment, the coesite sample was pressurized at room temperature up to $29 \mathrm{GPa}$ and then slowly heated up to $400{ }^{\circ} \mathrm{C}$. During heating up to $200{ }^{\circ} \mathrm{C}$ the pressure dropped down to $27 \mathrm{GPa}$ and was readjusted before further heating. In the second experiment, the coesite sample was pressurized from room pressure up to $34 \mathrm{GPa}$ and then slowly heated up to $200^{\circ} \mathrm{C}$. At this temperature, however, the Raman spectrum was very poor and therefore the experiment was interrupted. Pressure was monitored from a position of the ruby fluorescence peaks corrected for temperature after Rekhi et al. (1999).

\section{Transmission electron microscopy}

Conventional TEM characterizations in bright-field and dark-field imaging and selected electron diffraction techniques were carried out with a Philips CM20FEG equipped with an energy-dispersive X-ray (EDX) analyzer, operated at $200 \mathrm{kV}$. Analyses were performed on several chips of a crystal that was subjected to a maximum pressure of $35 \mathrm{GPa}$. The recovered material was first characterized by Raman spectroscopy and then further crushed between two tungsten carbide plates. The ethanol dispersion was placed on a lacey carbon film on $\mathrm{Cu}$-grid (300 mesh). Selected-area electron diffraction (SAED) patterns from different chips were obtained to distinguish between crystalline and amorphous domains or particles. The chemistry of the grains was also cross-checked using EDX spectroscopy.

\section{X-ray diffraction}

We selected coesite single crystals at BGI using a rotating anode high-brilliance Rigaku diffractometer with MoK $\alpha$ radiation, equipped with Osmic focusing X-ray optics and Bruker Apex CCD detector.

The crystal recovered after a repeated experiment at $34 \mathrm{GPa}$ and $\sim 300{ }^{\circ} \mathrm{C}$ was analyzed by means of single-crystal X-ray diffraction at the beamline 13IDD of the GSECARS at the Advanced Photon Source, Argonne National Laboratory, U.S.A. 


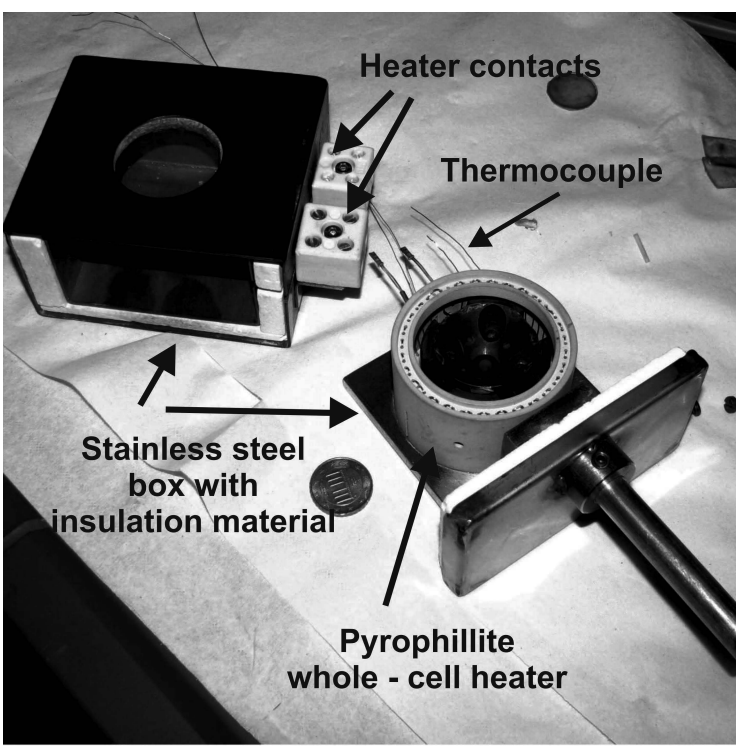

FIGURE 1. External heating assembly, consisting of a pyrophillite whole cell heater surrounding DAC placed on a stainless steel holder and double-layered stainless-steel insulation box. The coin is used as a scale marker.

An incident monochromatic beam with wavelength of $0.3344 \AA$ was focused to a spot of $4 \times 8 \mu \mathrm{m}^{2}$. Diffraction images were collected using a MAR165 CCD detector with sample-to-detector distance of approximately $250 \mathrm{~mm}$. The sample was also analyzed by X-ray diffraction mapping along $5 \times 5$ points, covering an area of $12 \times 12 \mu \mathrm{m}^{2}$ (step size $3 \mu \mathrm{m}$ ), with exposure time of $10 \mathrm{~s}$ at each point. Diffraction images were integrated using the Fit2d software (Hammersley 1998).

\section{Ab initio calculations}

We analyze the pressure dependence of the phonon spectra also from the firstprinciples calculations. We determine the ground-state properties using standard density-functional theory (Kohn and Sham 1965; Payne et al. 1992; Martin 2003) in the ABINIT implementation, based on planewaves and pseudopotentials (Gonze et al. 2002, 2009). Starting from the crystal structure refined by Angel et al. (2003) we determine the theoretical structure of coesite up to $40 \mathrm{GPa}$ in $10 \mathrm{GPa}$ steps. Then we compute the energy derivatives to build the dynamical matrices and the Raman tensors in the framework of the density-functional perturbation theory (Baroni et al. 2001; Gonze et al. 2005; Veithen et al. 2005). We employ a $4 \times 4 \times 4$ grid of special $\mathrm{k}$ points (Monkhorst and Pack 1976) to sample the electron density in the reciprocal space and a kinetic energy cutoff of 38 Hartrees $(1$ Hartree $=27.2116$ $\mathrm{eV})$. With these parameters the precision of the calculation is typical on the order of 0.001 Hartree in energy and better than $1 \mathrm{GPa}$ in pressure. We store all the Raman spectra computed under pressure on the WURM web site (http://www. wurm.info, archived at http://www.webcitation.org/6NAEO11G6). More details of the Raman calculations can be found in the original WURM paper (Caracas and Bobocioiu 2011).

\section{RESULTS}

\section{High-pressure, room-temperature Raman spectroscopy}

Selected Raman spectra collected during compression are shown in Figure 2. At ambient pressure coesite shows prominent peaks at 202, 261, 322, 352, 371, 425, 469, and $519 \mathrm{~cm}^{-1}$, with the last peak being the most intense. The intensities and positions of these peaks are in good agreement with previous studies (Sharma et al. 1981; Boyer et al. 1985; Hemley 1987). The peaks that are expected to be centered around 665 and 785 $\mathrm{cm}^{-1}$ at ambient pressure have only been observed at elevated pressures and not in all experiments. A peak located at $815 \mathrm{~cm}^{-1}$
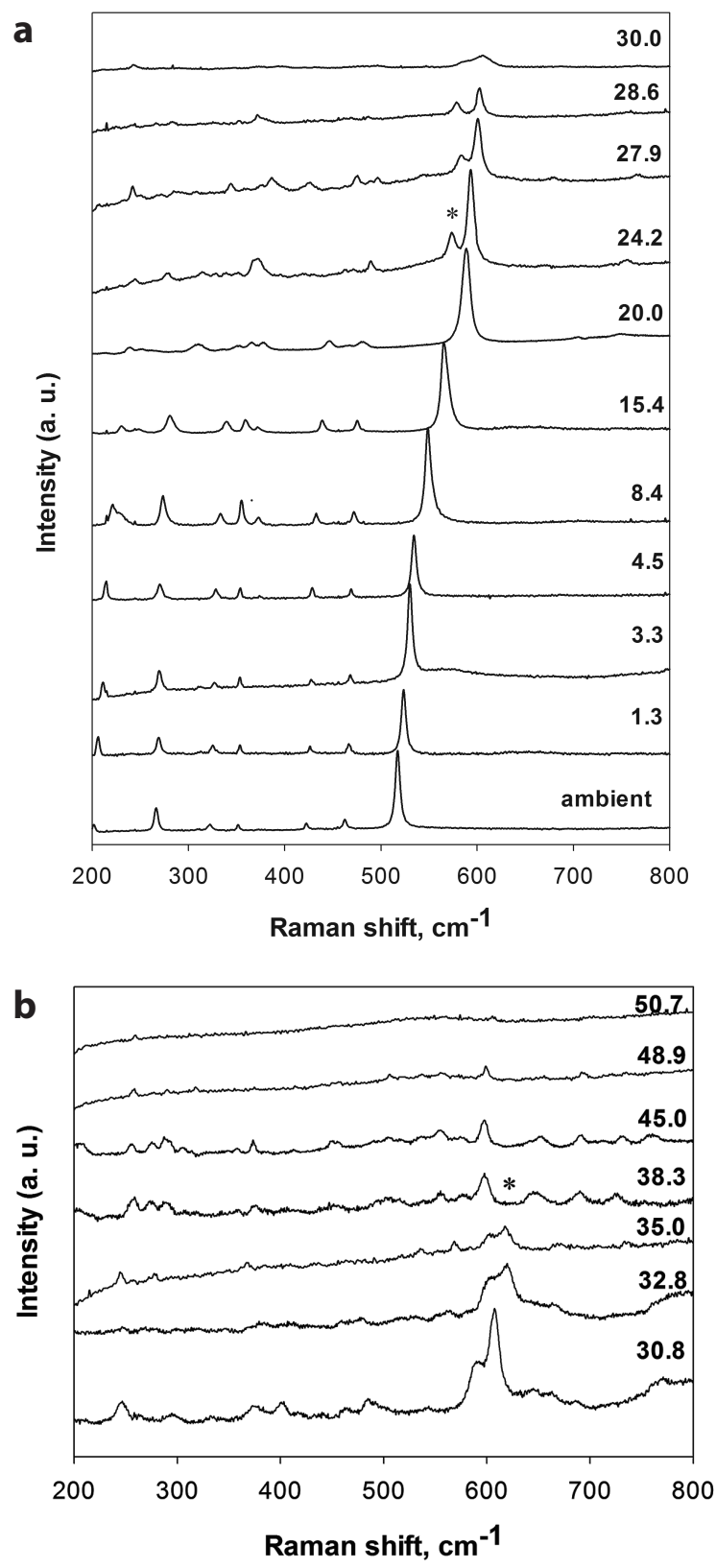

FIGURE 2. (a) Raman spectra collected up to $30 \mathrm{GPa}$ during compression. The star located above the doublet peak at $24.2 \mathrm{GPa}$ indicates appearance of coesite II phase. (b) Raman spectra collected between 30 and $51 \mathrm{GPa}$ during compression. The star above the spectrum at $38.3 \mathrm{GPa}$ indicates disappearance of the main doublet peak and the transition to coesite III.

was observed starting from ambient conditions, but was not detected in all experiments. Another peak, not previously reported in high-pressure Raman spectroscopy studies of coesite, was observed in two experiments. It appeared at and above 4.5 $\mathrm{GPa}$, and, according to its pressure shift, it can be expected to occur around $860 \mathrm{~cm}^{-1}$ at ambient conditions. Raman bands at 1036 and $1164 \mathrm{~cm}^{-1}$ were observed at ambient conditions outside the DACs, but not under compression due to their very 
low intensities. All the vibration bands are significantly weaker than the main $519 \mathrm{~cm}^{-1}$ band, they all show continuous positive pressure shift during compression and can be followed up to $\sim 28 \mathrm{GPa}$ (Fig. 2a). The bands located at 519, 665, 785, and 815 $\mathrm{cm}^{-1}$ have the largest pressure shifts, in good agreement with the previous study by Hemley (1987).

The most intense $519 \mathrm{~cm}^{-1}$ band was described as $v_{s}(\mathrm{Si}-\mathrm{O}-$ Si) symmetric stretching mode with $\mathrm{A}_{\mathrm{g}}$ symmetry (Sharma et al. 1981, and references therein). It shows continuous positive pressure increase up to $23 \mathrm{GPa}$. Above $23 \mathrm{GPa}$ a new strong vibrational band appears at a frequency of about $20 \mathrm{~cm}^{-1}$ lower than the main Raman peak, which at this pressure has a frequency of $593 \mathrm{~cm}^{-1}$, as was also observed by Hemley (1987). Several other new modes become visible at lower frequencies, confirming the results reported by Hemley (1987) and their interpretation of a possible phase transition to a phase with reduced symmetry or enlarged unit cell. This post-coesite phase, hereafter referred as coesite II, can be followed upon compression up to $\sim 35 \mathrm{GPa}$ (Fig. 2b). The $A_{g}$ mode persists up to $\sim 35 \mathrm{GPa}$, although its intensity decreases significantly. Above $\sim 35 \mathrm{GPa}$, the two highfrequency peaks disappear; only one new peak centered around $\sim 600 \mathrm{~cm}^{-1}$ can be observed (Fig. 2 b). This sudden change in the Raman spectrum indicates a possible discontinuous transition to a new phase, hereinafter referred as to coesite III. On further compression up to $\sim 51 \mathrm{GPa}$, the peak centered around $\sim 600 \mathrm{~cm}^{-1}$ decreases in intensity and exhibits a very small pressure increase (Fig. 2b). Above $\sim 35 \mathrm{GPa}$, in addition to the peak centered around $\sim 600 \mathrm{~cm}^{-1}$, new bands appear as two triplets of equally spaced peaks between $\sim 250-300 \mathrm{~cm}^{-1}$ and between $\sim 620-720$ $\mathrm{cm}^{-1}$. Appearance of a large number of new vibrational modes may indicate that coesite III has either an enlarged unit cell or lower symmetry with respect to coesite I and II structures. At $\sim 51 \mathrm{GPa}$ no vibrational modes can be observed. The pressure dependence of the main $519 \mathrm{~cm}^{-1}$ Raman mode $(d v / d P)$, where $v$ is the ambient-pressure phonon frequency and $P$ is the pressure) was derived from a series of experiments and it is, on average, 3.0 $\pm 0.4 \mathrm{~cm}^{-1} / \mathrm{GPa}$ (Fig. 3). Based on the zero-pressure bulk modulus $K_{T 0}=100.8 \pm 0.5 \mathrm{GPa}$ reported by Angel et al. (2001) and using the formulation of the mode-Grüneisen parameter as $\gamma \nu=\left(K_{T 0} / v\right)$ $(d v / d P)$, we obtain a value of $0.58 \pm 0.08$ for the $\mathrm{A}_{\mathrm{g}}$ mode, only slightly higher than the values reported in Hemley (1987).

At the highest pressure reached in these experiments, about $51 \mathrm{GPa}$, the Raman spectrum of coesite almost vanishes. Under decompression the vibrational modes of coesite III regain intensity, with the most prominent peaks visible at $\sim 250, \sim 540$, and $\sim 580 \mathrm{~cm}^{-1}$. Their frequencies decrease continuously down to $\sim 24$ GPa without any abrupt change, suggesting that the structure of coesite III is preserved down to $24 \mathrm{GPa}$ and that the transition to the coesite II phase is not reversible or has a very large hysteresis. A strong single band centered around $600 \mathrm{~cm}^{-1}$ appears at $\sim 24$ $\mathrm{GPa}$ and its intensity increases with further decompression, until it dominates the Raman spectra below $\sim 20 \mathrm{GPa}$, suggesting that the structure of coesite has been retrieved. Indeed, the Raman spectrum of the recovered crystal at room pressure is identical to that of the starting material (Fig. 4). The fact that the pressurequenched material is crystalline coesite strongly suggests that the high-pressure phase coesite III was also crystalline at $51 \mathrm{GPa}$ even if the Raman vibrations could not be observed.

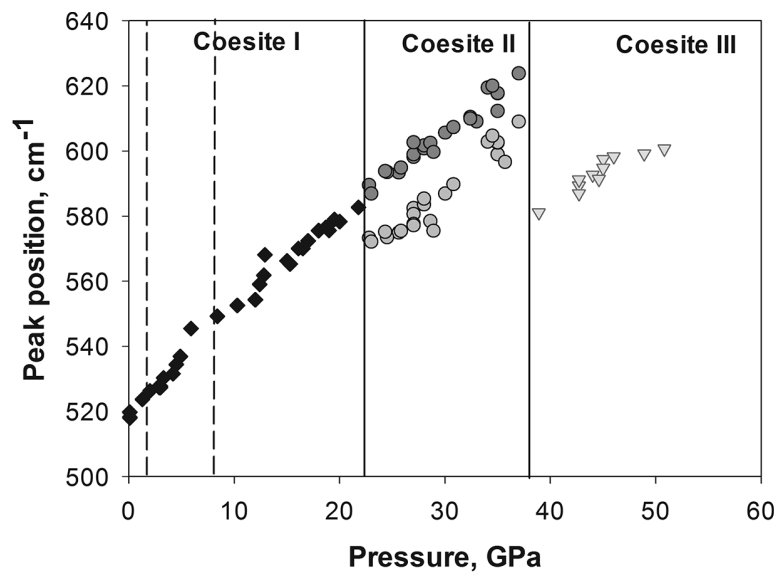

FIGURE 3. Pressure dependence of the main Raman active mode obtained from several different experiments at ambient temperature. Phase transitions at $\sim 23$ and $\sim 35 \mathrm{GPa}$ are indicated by solid lines, and the thermodynamic stability field of coesite is indicated by dotted lines.

\section{Raman active modes from ab initio calculations}

We compute the phonons in the Brillouin zone-center, with both frequencies and atomic displacement patterns, for coesite I up to $40 \mathrm{GPa}$. We equally determine the Raman tensors and compute the Raman intensities for the Raman-active modes. All the spectra are reported on the WURM web site (see http://www. webcitation.org/6NAEO11G6), but for sake of clarity only the spectrum at ambient pressure is represented here, in Figure 5a. Figure $5 \mathrm{~b}$ shows pressure dependence of selected most dominant modes. The Raman spectra are dominated by the $\mathrm{A}_{\mathrm{g}}$ mode situated at $\sim 510 \mathrm{~cm}^{-1}$ at ambient pressure. A series of low-intensity peaks are observed at frequencies higher than $600 \mathrm{~cm}^{-1}$, in excellent agreement with the experimental data.

The strongest $\mathrm{A}_{\mathrm{g}}$ mode, located at $\sim 510 \mathrm{~cm}^{-1}$ at ambient pressure (at $541 \mathrm{~cm}^{-1}$ at $10 \mathrm{GPa}$ ) is a breathing mode of the four-membered rings comprised of $\mathrm{SiO}_{4}$ tetrahedra (Fig. 6a)

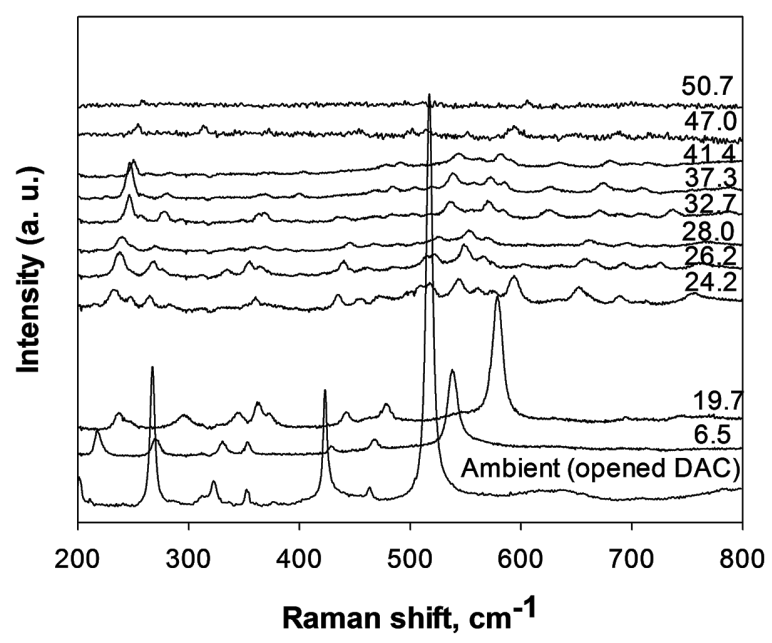

FIGURE 4. Raman spectra collected during decompression from $\sim 51$ $\mathrm{GPa}$ to ambient pressure. Note that the power of the incident laser beam was decreased below $20 \mathrm{GPa}$ from 1.0 to $0.3 \mathrm{~W}$. 
and it arises from bending of four different $\mathrm{Si}-\mathrm{O}-\mathrm{Si}$ bonds that vibrate in phase, namely $\mathrm{Si}-\mathrm{O} 2-\mathrm{Si}$, Si-O3-Si, Si-O4-Si, and SiO5-Si (nomenclature after Angel et al. 2003, see Figs. 2 and 4 therein). Except for the Si-O4-Si bond angle, which changes by only a few degrees throughout the pressure range examined by Angel et al. (2003), other three bond angles show strong pressure dependence. In contrast to these four angles, Si-O1-Si is rigid due to symmetry constrains and remains $180^{\circ}$ throughout the investigated range. Atomic movements of this breathing $\mathrm{A}_{\mathrm{g}}$ mode are illustrated in a representative four-membered ring in Figure 6a seen during contraction. The largest displacement is seen in $\mathrm{O} 3$ and $\mathrm{O} 4$ atoms, which are moving symmetrically inward, and in $\mathrm{O} 5$ atoms, that are moving parallel to each other along $a$-axes, but in the opposite direction.

A $B_{\mathrm{g}}$ mode that appears at $77 \mathrm{~cm}^{-1}$ at ambient conditions (also $77 \mathrm{~cm}^{-1}$ at $10 \mathrm{GPa}$ and $56 \mathrm{~cm}^{-1}$ at $20 \mathrm{GPa}$ ) is dominant in the lower-frequency region and shows softening with increasing pressure (Fig. 5b), similar to previous experimental studies (Hemley 1987). This mode corresponds to shearing of the tetrahedra rings (Figs. 6b and 6c) and may have a significant influence on the structural distortion at elevated pressures. Shearing of the four-membered rings is illustrated by the two adjacent four-membered rings shown in Figure 6b. Within the same ring $\mathrm{O} 3$ atoms are displaced in the same direction, but this displacement direction is opposite between the two adjacent rings. $\mathrm{O} 2$ atoms vibrate opposite to the $\mathrm{O} 3$ displacement direction. Within the ring, one of the $\mathrm{O} 4$ atoms moves downward and the other upward from the plane of the ring. All O5 atoms of the structure move in the same direction, in the plane of the $\mathrm{SiO}_{4}$ rings. Similar intensity variation is observed in an $\mathrm{A}_{\mathrm{u}}$ mode that appears at $115 \mathrm{~cm}^{-1}$ at ambient pressure (Fig. 5b). Above $20 \mathrm{GPa}$ this infrared active mode becomes even more unstable than the Raman active $77 \mathrm{~cm}^{-1}$ mode; this enhances the displacement of the $\mathrm{O} 1$ atom from the rigid $180^{\circ} \mathrm{Si}-\mathrm{O} 1-\mathrm{Si}$ bond (Fig. 6d). Then this displacement could initiate the structural changes that we observe above $24 \mathrm{GPa}$. However, an IR study is required to confirm this prediction. For a better visualization of the atomic motions, please visit the WURM web site.

Other two modes in the $400-500 \mathrm{~cm}^{-1}$ region (Fig. 5a) are correlated with the motion of the $\mathrm{SiO}_{4}$ tetrahedra rings, although they have low intensity. One is a complex $\mathrm{B}_{\mathrm{g}}$ mode at $430 \mathrm{~cm}^{-1}$, corresponding to both breathing and shearing of the $\mathrm{SiO}_{4}$ tetrahedra rings, and the other one is an $\mathrm{A}_{\mathrm{g}}$ mode at $455 \mathrm{~cm}^{-1}$, which arises from stretching of the rings. The $430 \mathrm{~cm}^{-1}$ mode has not been observed experimentally, but the $455 \mathrm{~cm}^{-1}$ mode, albeit very weak, was present in some of our spectra.

\section{High-temperature, high-pressure Raman spectra}

The Raman spectra collected at 27-29 GPa up to $400{ }^{\circ} \mathrm{C}$ and at $34 \mathrm{GPa}$ up to $200{ }^{\circ} \mathrm{C}$ are shown in Figures $7 \mathrm{a}$ and $7 \mathrm{~b}$, respectively. The first heating experiment was carried out in the pressure range where coesite II phase (Figs. 2a and $2 \mathrm{~b}$ ) was observed, and, as expected, the main feature in the Raman spectra is the peak doublet located $\sim 600 \mathrm{~cm}^{-1}$ (Fig. 7a). The intensity of the vibrational modes decreases with increasing temperature, and at $27 \mathrm{GPa}$, as expected, they shift to lower frequency. However, at $29 \mathrm{GPa}$ the higher frequency peak shifted $4 \mathrm{~cm}^{-1}$ toward higher frequency between room temperature and $425^{\circ} \mathrm{C}$, whereas the lower-frequency band softened of $\sim 1 \mathrm{~cm}^{-1}$, increasing in this way the total spread of the doublet. At this temperature an intermediate peak appears in the spectra but due to the very poor quality of the spectra it is hard to tell if it is a signal from the sample or a spike. At higher temperatures the intensity of the doublet decreased significantly and the doublet peak was not observable.

At $34 \mathrm{GPa}$ and $100{ }^{\circ} \mathrm{C}$, the peak doublet of coesite II is still present and decreases both in intensity and in frequency with
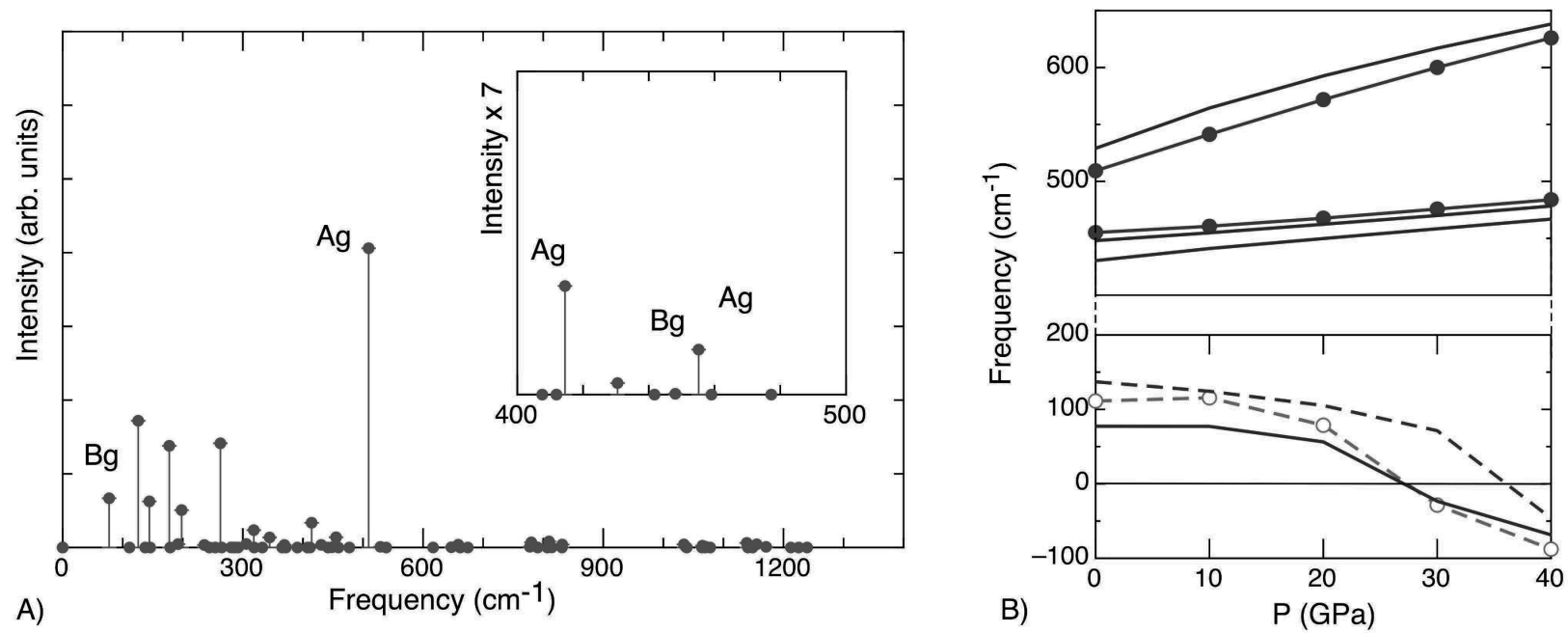

FigURE 5. (a) Theoretical Raman spectrum at ambient conditions. The spectrum is dominated by the strong $A_{\mathrm{g}}$ mode at $510 \mathrm{~cm}^{-1}$. Several peaks are visible at low frequencies and the most intense is the $\mathrm{B}_{\mathrm{g}}$ mode at $77 \mathrm{~cm}^{-1}$, while all peaks above $600 \mathrm{~cm}^{-1}$ are very weak. A few peaks are symmetry-labeled. The inset shows the peaks occurring in the $400-500 \mathrm{~cm}^{-1}$ range, with intensity magnified, with the $B_{\mathrm{g}}$ mode at 430 and the $\mathrm{A}_{\mathrm{g}}$ mode at $455 \mathrm{~cm}^{-1}$. (b) Pressure variation of selected modes in coesite. Note that the lowest frequency modes become unstable with increasing pressure. The A modes bear symbols; the g modes are represented with solid line; the dashed line represent modes that are only infrared active. Three modes become unstable between 20 and $30 \mathrm{GPa}$, including a $\mathrm{B}_{\mathrm{g}}$ Raman active mode at $77 \mathrm{~cm}^{-1}$ and an $\mathrm{A}_{\mathrm{u}}$ infrared active at $111 \mathrm{~cm}{ }^{-1}$ (ambient conditions). These two modes are displayed in Figures $6 \mathrm{c}$ and $6 \mathrm{~d}$ at elevated pressure. 


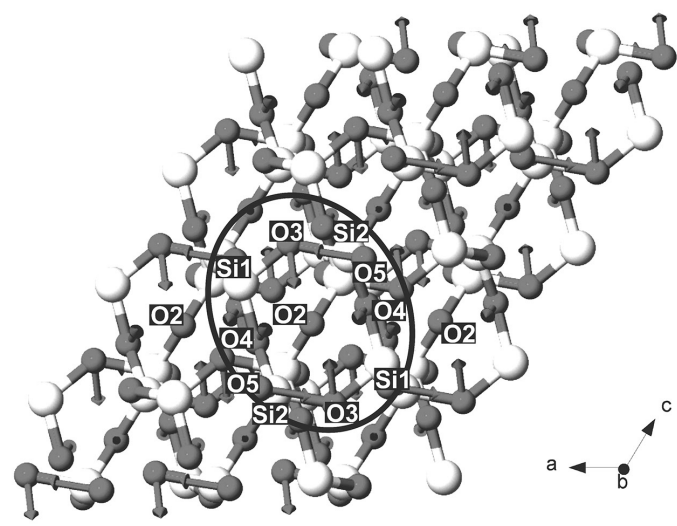

a
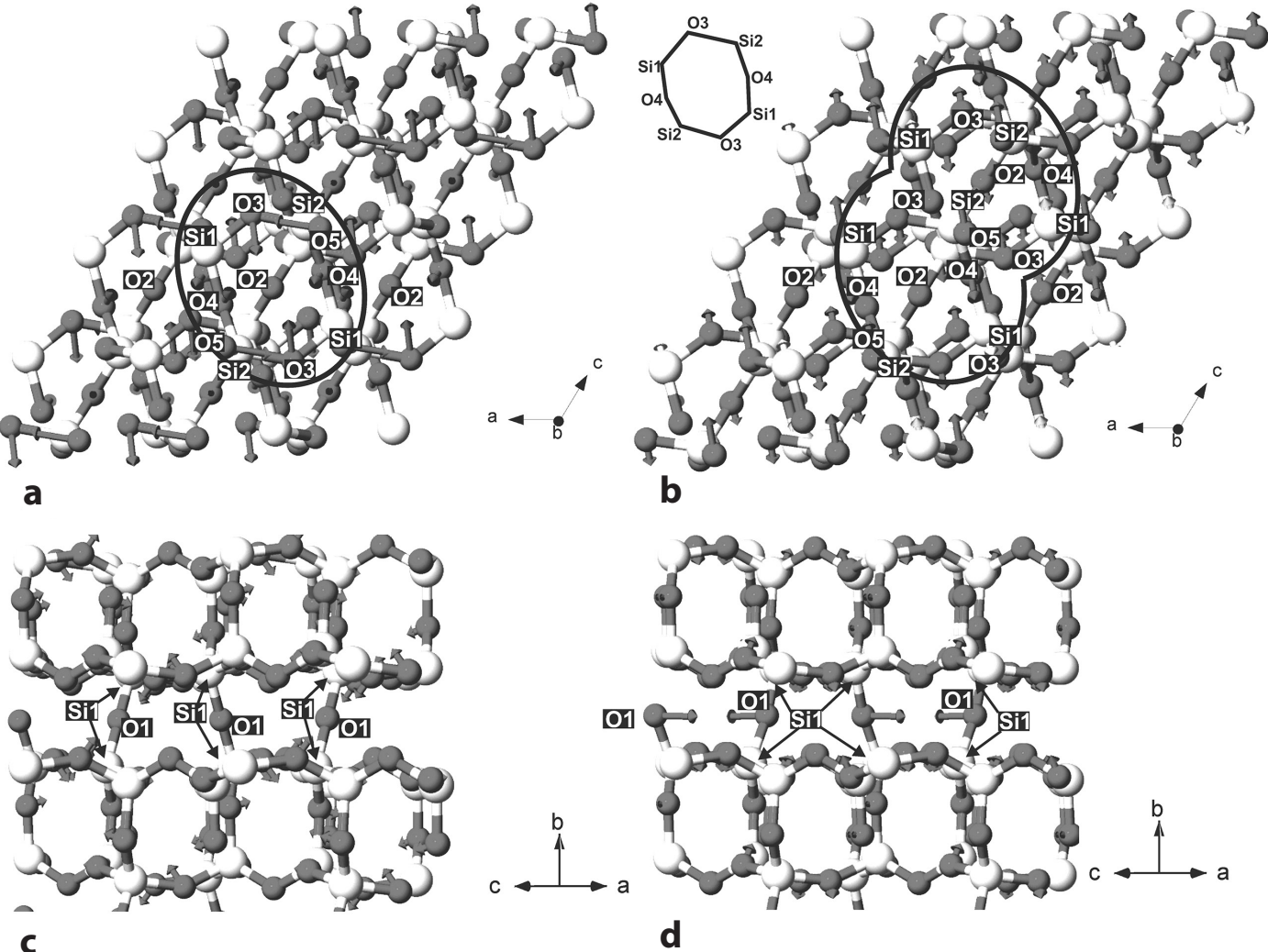

FIGURE 6. Atomic displacement pattern of some of the most representative Raman modes of coesite at $10 \mathrm{GPa}$. They dominate the Raman spectra and their behavior is likely responsible for the structural changes exhibited under pressures. White corresponds to silicon atom, gray is oxygen; atomic nomenclature is after Angel et al. (2003). Ellipses are surrounding representative $\mathrm{SiO}_{4}$ rings and gray arrows placed on oxygen atoms are indicating displacement directions. (a) The strongest $\mathrm{A}_{\mathrm{g}}$ mode at $541 \mathrm{~cm}^{-1}$ (located at $510 \mathrm{~cm}^{-1}$ at ambient pressure) is a breathing mode of the four-membered tetrahedral rings. (b) The $\mathrm{B}_{\mathrm{g}}$ mode at $77 \mathrm{~cm}^{-1}$ (also $77 \mathrm{~cm}^{-1}$ at $10 \mathrm{GPa}$ ) dominates the spectra at low frequencies and it corresponds to shearing of the four-membered silica rings. The sketch in the upper left corner on the figure is for simplifying the structure of one ring. (c) The same $\mathrm{B}_{\mathrm{g}}$ mode at $20 \mathrm{GPa}\left(56 \mathrm{~cm}^{-1}\right)$ in different crystallographic orientation. (d) Infrared active $\mathrm{A}_{\mathrm{u}}$ mode at $20 \mathrm{GPa}\left(78 \mathrm{~cm}^{-1}\right)$ showing significant displacement of the $\mathrm{O} 1$ atom from the rigid $180^{\circ} \mathrm{Si}-\mathrm{O} 1-\mathrm{Si}$ bond.
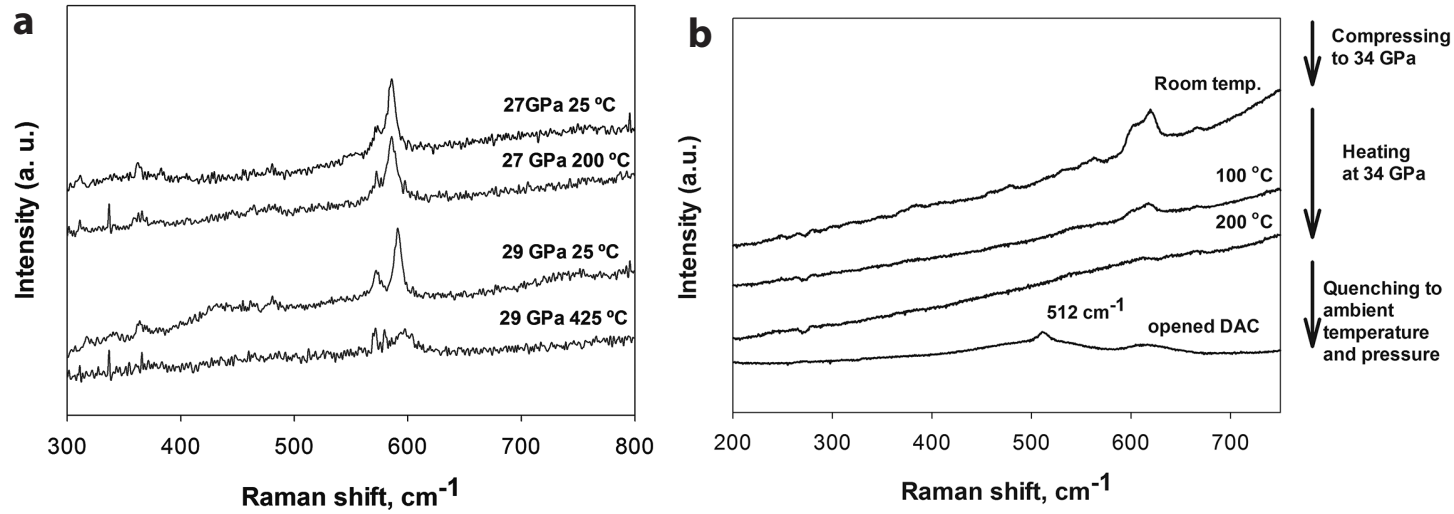

FIGURE 7. (a) Raman spectra collected at 27-29 GPa during heating up to $400{ }^{\circ} \mathrm{C}$. At both pressures one can notice a decrease in intensity and an increase in the splitting of the doublet. (b) Raman spectra collected at $34 \mathrm{GPa}$ during heating up to $200{ }^{\circ} \mathrm{C}$ and after quenching to ambient conditions. Only a decrease in intensity can be observed at this pressure, without an increase in the splitting of the doublet. 
increasing temperature. No signal was observed at $200^{\circ} \mathrm{C}$ (Fig. 7b). Raman spectra were collected during decompression at elevated temperature, but no Raman spectra of coesite were observed. At room pressure and after cooling down to room temperature, a broad peak close in position to the main vibration mode of coesite was observed (Fig. 7b). The recovered crystal was also analyzed by single-crystal X-ray diffraction (see further).

\section{TEM observations}

One of the recovered samples was investigated by transmission electron microscopy. This sample was selected for the TEM analysis because the central, thickest part of the crystal was bridged between diamond anvils at $\sim 35 \mathrm{GPa}$ during compression, whereas the thinner edges of the crystal remained largely undisturbed by the diamond culets. Raman spectra collected on the material recovered from the thin edge of the sample show a weak peak around 425 and $514 \mathrm{~cm}^{-1}$, and the TEM analysis of the same material revealed that very thin grain domains are indexed with coesite in the SAED pattern (Fig. 8). On the other hand, the material recovered from the bridged part of the crystal does not show any Raman active modes and appears entirely amorphous at the TEM. Our observations agree qualitatively with the previous studies on high-pressure silica behavior: in experiments with stiff pressure media where amorphization is initiated at lower pressures with respect to experiments in quasihydrostatic conditions (e.g., Hemley 1987; Haines et al. 2001).

\section{X-ray diffraction}

$\mathrm{X}$-ray mapping along 25 spots of the sample quenched from $\sim 300{ }^{\circ} \mathrm{C}$ and $34 \mathrm{GPa}$ indicates that the material is well crystallized. All observed diffraction peaks belong to coesite (Fig. 9). Phase identification was carried out using the Match! Software v.1.11 (with Crystallography Open Database), considering only the region of $d$-spacing between 4.5 and $1.0 \AA\left(2 \theta 5-19^{\circ}\right)$. The suggested possible phases by the program are coesite, diamond, and quartz; however, all of the peaks assigned to quartz have $d$-spacing that can also be assigned to coesite, and no peak belonging solely to quartz has been observed (Fig. 9).

\section{DISCUSSION}

Coesite appears to undergo at least two distinct transitions to metastable phases upon compression, as shown in DAC experiments with quasi-hydrostatic medium $(\mathrm{Ne})$ up to $~ 51 \mathrm{GPa}$. A similar high-pressure behavior, i.e., complex path of transformations to metastable phases before amorphization, have been reported for quartz (Kingma et al. 1993; Haines et al. 2001), and it is in agreement with predictions from numerical models, which suggest that several metastable phases can appear on compression of $\mathrm{SiO}_{2}$ materials before amorphization (Wentzcovitch et al. 1998; Dubrovinsky et al. 2004). Most certainly these phases appear as a consequence of the lack of energy at room temperature to overcome the considerable potential barrier needed to increase the silicon coordination by oxygen from 4 to 6 .

The transition from coesite I to coesite II at around $23 \mathrm{GPa}$ was initially reported by Hemley (1987). The study focused on the phase transition, showing its relation to phonon softening, but did not go further into clarifying the eventual splitting of the

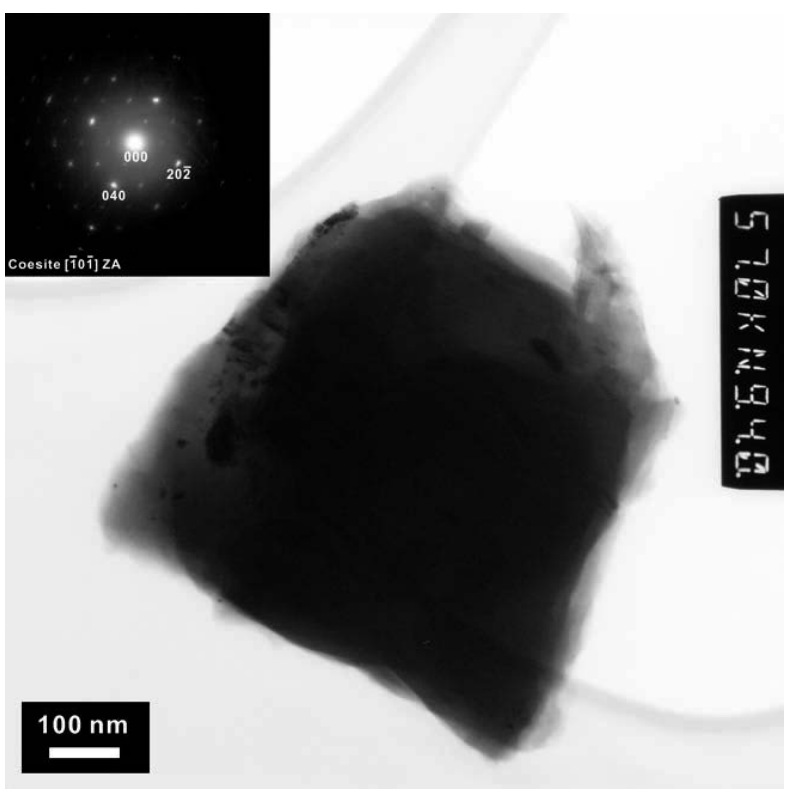

FIGURE 8. TEM analysis of one of the grains recovered from the thin edge of the crystal bridged at $35 \mathrm{GPa}$, showing diffraction profiles that can be attributed to coesite. The material from which this grain was selected shows a weak Raman peak around $520 \mathrm{~cm}^{-1}$.

most intense Raman doublet above $\sim 23 \mathrm{GPa}$. The author also reports one single broad peak above $30 \mathrm{GPa}$ centered around $600 \mathrm{~cm}^{-1}$, interpreted as a feature similar to that reported for silica glass, and therefore concludes that above such pressure coesite becomes amorphous. Hemley (1987) also reported that the splitting at $23 \mathrm{GPa}$ was obscured in all experiments except in those with argon, which was providing the best quasi-hydrostatic conditions in their experiments at higher pressures. Neon is shown to be a softer pressure-transmitting medium and to give quasi-hydrostatic conditions at higher pressures than argon (Klotz et al. 2009). In our experiments, therefore, we are able not only to clearly follow the evolution of the splitting of the main Raman mode above $23 \mathrm{GPa}$, but also to observe another

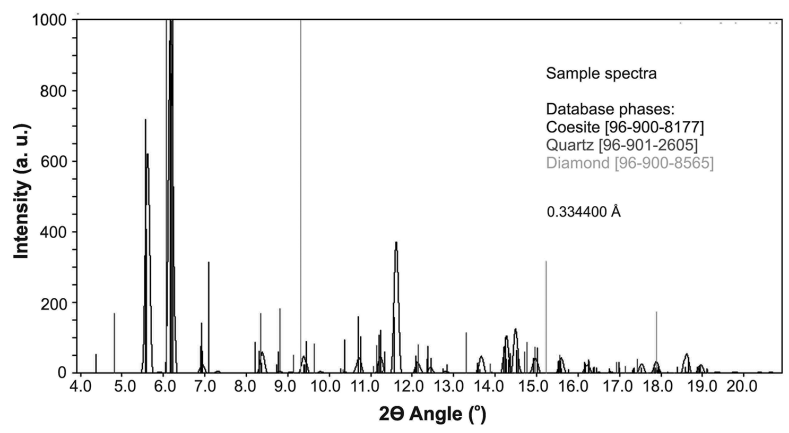

FIGURE 9. Representative X-ray diffraction data showing that the crystalline phase quenched from $\sim 34 \mathrm{GPa}$ and $\sim 300{ }^{\circ} \mathrm{C}$ is coesite. Reference phases are taken from Crystallography Open Database: coesite $=$ Araki and Zoltai (1969), synthehic sample at ambient conditions; quartz = Hazen et al. (1989), synthetic sample at pressure of 1 bar; and diamond $=$ Wyckoff $(1963)$. 
phase transition at $\sim 35 \mathrm{GPa}$. Non-hydrostatic compression, as we observed on the example of partially bridged coesite crystal as well as reported in the experiments with Ar or stiffer pressure media (Hemley 1987), leads to amorphization of coesite at lower pressures at room temperature.

Our experimental and theoretical results are in very good agreement, with a discrepancy in frequencies of about $10 \mathrm{~cm}^{-1}$ between the calculated and the measured spectra. The main Raman mode is at $519 \mathrm{~cm}^{-1}$ in the experimental spectra and at 510 $\mathrm{cm}^{-1}$ in the calculated; another intense $\mathrm{A}_{\mathrm{g}}$ mode that is observed at $426 \mathrm{~cm}^{-1}$ is calculated at $415 \mathrm{~cm}^{-1}$. Moreover, the calculated frequency shifts with pressure are very similar to those observed experimentally, at least for the strongest Raman modes.

At $8 \mathrm{GPa}$, a small shoulder at $228 \mathrm{~cm}^{-1}$ (Fig. 2a) can be observed overlying the $220 \mathrm{~cm}^{-1}$ peak $\left(202 \mathrm{~cm}^{-1}\right.$ at ambient pressure). From a previous study (Hemley 1987) it appears that the mode at $176 \mathrm{~cm}^{-1}$ intersects the $202 \mathrm{~cm}^{-1}$ mode at $\sim 8 \mathrm{GPa}$, and therefore it could be the cause of the shoulder in the spectra we recorded at this pressure. However, it should be noted that the results of our ab initio calculations are in contrast with such an interpretation since these two calculated modes do not cross at any pressure (calculated up to $40 \mathrm{GPa}$ ), although their separation decreases with increasing pressure.

In light of our experiments and ab initio calculation we can give an insight on the mechanism of transformation from the coesite I to coesite II phase in terms of a complex phonon softening. The crystal structure of coesite II has a lower symmetry than coesite I, as it appears from the increasing number of the vibrational modes observed in this study. The calculations show that three phonon modes, one $A_{g}$, one $A_{u}$, and one $B_{u}$, soften under compression (Fig. 4b), the former two becoming imaginary around $27 \mathrm{GPa}$. They all correspond to some form of shear of the $\mathrm{SiO}_{4}$ tertrahedra rings, but the $\mathrm{A}_{u}$ and $\mathrm{B}_{\mathrm{u}}$ modes are only infrared active. Freezing of any or all of these modes could enhance a displacive transition and may be associated with a breaking of the symmetry of the four-membered rings. This can also give rise to two slightly different breathing modes for two differently sheared rings, a consequence of which is the splitting of the main $\mathrm{A}_{\mathrm{g}}$ mode (at $510 \mathrm{~cm}^{-1}$ at room pressure) associated with the breathing of the four-membered rings.

An alternative explanation is that the second Raman peak at $560 \mathrm{~cm}^{-1}$ in the coesite II structure arises from the coupling between the soft $\mathrm{B}_{\mathrm{g}}$ mode, situated at $77 \mathrm{~cm}^{-1}$ at ambient conditions and imaginary at the transition, with either the $\mathrm{B}_{\mathrm{g}}$ mode at $430 \mathrm{~cm}^{-1}$ (at ambient conditions, $450 \mathrm{~cm}^{-1}$ at $20 \mathrm{GPa}$ ) or the $\mathrm{A}_{\mathrm{g}}$ mode at $455 \mathrm{~cm}^{-1}$ (at ambient conditions, $468 \mathrm{~cm}^{-1}$ at $20 \mathrm{GPa}$ ). In particular the coupling and further energy transfer between the two $\mathrm{B}_{\mathrm{g}}$ modes is very likely due to their identical symmetry. This transfer can also enhance the Raman intensity of the higherenergy mode. Coupling of the unstable $\mathrm{B}_{\mathrm{g}}$ and the stable $\mathrm{A}_{\mathrm{g}}$ modes is possible because of similarities between their eigendisplacements. The coupling could also enhance the polarizability of the higher-energy mode and hence its Raman signature.

The transition from coesite I to coesite II is likely to be second order in character because of the phonon softening, continuous behavior of the main Raman peak, and because of the absence of hysteresis. Decrease in intensity and softening of the main peak doublet during heating, support the observation that coesite II is a metastable phase, which exists only at room temperatures far outside the pressure stability field of coesite. The heating that is insufficient to transform coesite to the thermodynamically stable phase, stishovite, enhances the thermal motion of the metastable structure and causes instability but, most probably, does not lead to amorphization. Hence, the pressure- and temperature-quenched sample retransforms to coesite. The anomalous spreading of the doublet and shifting to the higher frequency at $29 \mathrm{GPa}$ and $400{ }^{\circ} \mathrm{C}$ requires further understanding and structural analysis of coesite II.

The transition of coesite II to coesite III is possibly first order in character, as it appears from the abrupt change in Raman spectra above $\sim 35 \mathrm{GPa}$ (Fig. 2b). As expected in a first-order transition, the frequency of the main mode, situated at $\sim 600$ $\mathrm{cm}^{-1}$, does not vary significantly with pressure (Fig. 4) and a hysteresis is probably hindering reversible transformation from coesite III to coesite II.

The mechanisms of this transition cannot be revealed from $\mathrm{ab}$ initio calculation, since the structure of the two polymorphs is still unknown. If we assume that the major peak observed in the Raman spectra is still correlated to the O-Si-O motions as the $\mathrm{A}_{\mathrm{g}}$ mode, the major decrease in frequency may suggest a softer $\mathrm{Si}-\mathrm{O}$ bond and, hence an increase in coordination number of the Si. Coesite III would therefore have at least some of Si in octahedral coordination. However, to confirm such hypothesis, a structural study is required.

\section{IMPLICATIONS}

The study of the high-pressure behavior of coesite reveals two high-pressure polymorphs that remain crystalline far above the previously reported amorphization pressure. This significant discrepancy between previous and our current results implies that the metastable phase diagram of silica phases requires a substantial revision. Crystalline or amorphous metastable phases derived from coesite under high-pressure conditions can be of interest in geological application as potential tracers of peak transient pressure conditions reached in processes such as impacts or faulting.

\section{ACKNOWLEDGMENTS}

We very much appreciate the assistance of Stefan Übelhack, who manufactured the insulation box for our experiments. Hubert Schulze is gratefully acknowledged for the sample preparation and Sven Linhardt for his help in experiments with electrical heating. We are grateful to Andreas Audétat for providing the starting glass material and helping us analyze it using LA-ICP-MS. This work was funded as a part of Bavarian Elite Network (ENB) Graduate Program "Oxides." The firstprinciples calculations were done on the jade machine of CINES, under DARI grant x2013106368. Portions of this research were carried out at the light source PETRA III at DESY, a member of the Helmholtz Association (HGF). We acknowledge the support of GeoSoilEnviroCARS (Sector 13), which is supported by the National Science Foundation, Earth Sciences (EAR-1128799), and the Department of Energy, Geosciences (DE-FG02-94ER14466). All authors are thankful for the useful comments of Joseph Smyth and the anonymous reviewer, which helped us improve the quality of the manuscript.

\section{REFERENCES CITED}

Angel, R.J., Mosenfelder, J.L., and Shaw, C.S.J. (2001) Anomalous compression and equation of state of coesite. Physics of the Earth and Planetary Interiors, $124,71-79$

Angel, R.J., Shaw, C.S.J., and Gibbs, G.V. (2003) Compression mechanisms of coesite. Physics and Chemistry of Minerals, 30, 167-176.

Araki, T., and Zoltai, T. (1969) Refinement of a coesite structure. Zeitschrift für Kristallographie, 129, 381-387.

Baroni, S., de Gironcoli, S., Dal Corso, A., and Giannozzi, P. (2001) Phonons and related crystal properties from density-functional perturbation theory. Reviews in Modern Physics, 73, 515-562. 
Boyer, H., Smith, D.C., Chopin, C., and Lasnier, B. (1985) Raman microprobe (RMP) determinations of natural and synthetic coesite. Physics and Chemistry of Minerals, 12, 45-48.

Bromiley, G.D., and Keppler, H. (2004) An experimental investigation of hydroxyl solubility in jadeite and Na-rich clinopyroxenes. Contributions to Mineralogy and Petrology, 147, 189-200.

Caracas, R., and Bobocioiu E. (2011) The WURM project: A freely available web-based repository of computed physical data for minerals. American Mineralogist, 96, 437-443.

Chao, E.C.T., Shoemaker, E.M., and Madsen, B.M. (1960) First natural occurrence of coesite. Science, 132, 220-222.

Chopin, C. (1984) Coesite and pure pyrope in high-grade blueschists of the Western Alps: a first record and some consequences. Contributions to Mineralogy and Petrology, 86, 107-118.

Coes, L. (1953) A new dense crystalline silica. Science, 118, 131-132.

Dean, D.W., Wentzcovitch, R.M., Keskar, N., Chelikowsky, J.R., and Binggeli, N. (2000) Pressure-induced amorphization in crystalline silica: Soft phonon modes and shear instabilities in coesite. Physical Review B, 61, 3303-3309.

Dera, P., Lazarz, J.D., Prakapenka, V.B., Barkley, M., and Downs, R.T. (2011) New insights into the high-pressure polymorphism of $\mathrm{SiO}_{2}$ cristobalite. Physics and Chemistry of Minerals, 38, 517-529.

Dubrovinsky, L., Dubrovinskaia, N., Prakapenka, V., Seifert, F., Langenhorst, F., Dmitriev, V., Weber, H.-P., and Le Bihan, T. (2004) A class of new high-pressure silica polymorphs. Physics of the Earth and Planetary Interiors, 143-144, 231-240.

Glinnemann, J., King, H.E., Schulz, H., Hahn, T., La Placa, S.J., and Dacol, F. (1992) Crystal structures of the low-temperature quartz-type phases of $\mathrm{SiO}_{2}$ and $\mathrm{GeO}_{2}$ at elevated pressure. Zeitschrift für Kristallographie, 198, 177-212.

Gonze, X., Beuken, J.-M., Caracas, R., Detraux, F., Fuchs, M., Rignanese, G.-M., Sindic, L., Verstraete, M., Zerah, G., Jollet, F., and others. (2002) First-principle computation of material properties the ABINIT software project. Computational Materials Science, 25, 478-492.

Gonze, X., Rignanese, G.-M., and Caracas, R. (2005) First-principles studies of the lattice dynamics of crystals, and related properties. Zeitschrift für Kristallographie, 220, 458-472.

Gonze, X., Amadon, B., Anglade, P.-M., Beuken, J.-M., Bottin, F., Boulanger, P., Bruneval, F., Caliste, D., Caracas, R., Côté, M., and others. (2009) ABINIT: First-principles approach to material and nanosystem properties. Computer Physics Communications, 180, 2582-2615.

Haines, J., Léger, J.M., Gorelli, F., and Hanfland, M. (2001) Crystalline post-quartz phase in silica at high pressure. Physical Review Letters, 87, 155503.

Hammersley, J. (1998) FIT2D V9.129 Reference Manual V3.1. European Synchrotron Radiation Facility, ESRF International Report No. ESRF98HAO1T.

Hazen, R.M., Finger, L.W., Hemley, R.J., and Mao, H.K. (1989) High-pressure crystal chemistry and amorphization of alpha-quartz. Solid State Communications, 72, 507-511.

Hemley, R.J. (1987) Pressure dependence of Raman spectra of $\mathrm{SiO}_{2}$ polymorphs: $\alpha$-quartz, coesite, and stishovite. In M.H. Manghnani and Y. Syono, Eds., High-Pressure Research in Mineral Physics, p. 347-359. Wiley/Terrapub, Tokyo.

Kantor, I., Prakapenka, V., Kantor, A., Dera, P., Kurnosov, A., Sinogeikin, S., Dubrovinskaia, N., and Dubrovinsky, L. (2012) BX90: A new diamond anvil cell design for X-ray diffraction and optical measurements. Review of Scientific Instruments, 83, 125102.

Kingma, K.J., Hemley, R.J., Mao, H., and Veblen, D.R. (1993) New high-pressure transformation in $\alpha$-quartz. Physical Review Letters, 70, 3927-3930.

Klotz, S., Chervin, J.-C., Munsch, P., and Le Marchand, G. (2009) Hydrostatic limits of 11 pressure transmitting media. Journal of Physics D-Applied Physics, 42, 075413.

Koch-Müller, M., Fei, Y., Hauri, E., and Liu, Z. (2001) Location and quantitative analysis of $\mathrm{OH}$ in coesite. Physics and Chemistry of Minerals, 28, 693-705.

Kohn, W., and Sham, L.J. (1965) Self-consistent equations including exchange and correlation effects. Physical Review, 140, A1133-A1138.

Korsakov, A.V., Hutsebaut, D., Theunissen, K., Vandenabeele, P., and Stepanov, A.S. (2007) Raman mapping of coesite inclusions in garnet from the Kokchetav Massif (Northern Kazakhstan). Spectrochimica Acta Part A: Molecular and Biomolecular Spectroscopy, 68, 1046-1052.
Kurnosov, A., Kantor, I., Boffa-Ballaran, T., Lindhardt, S., Dubrovinsky, L., Kuznetsov, A., and Zehnder, B.H. (2008) A novel gas-loading system for mechanically closing of various types of diamond anvil cells. Review of Scientific Instruments, 79, 045110.

Mao, H.K., Xu, J., and Bell, P.M. (1986) Calibration of the ruby pressure gauge to 800 kbar under quasi-hydrostatic conditions. Journal of Geophysical Research, 91, 4673-4676.

Martin, R.M. (2003) Electronic Structure. Basic theory and practical methods, 596 pp. Cambridge University Press, U.K.

Monkhorst, H.J., and Pack, J.D. (1976) Special points for Brillouin-zone integrations. Physical Review B, 13, 5188-5192.

Mosenfelder, J.L. (2000) Pressure dependence of hydroxyl solubility in coesite. Physics and Chemistry of Minerals, 27, 610-617.

Mosenfelder, J., and Bohlen, S. (1997) Kinetics of the coesite to quartz transformation. Earth and Planetary Science Letters, 153, 133-147.

Ohtani, E., Ozawa, S., Miyahara, M., Ito, Y., Mikouchi, T., Kimura, M., Arai, T., Sato, K., and Hiraga, K. (2011) Coesite and stishovite in a shocked lunar meteorite, Asuka-881757, and impact events in lunar surface. Proceedings of the National Academy of Sciences, 108, 463-466.

Parkinson, C.D. (2000) Coesite inclusions and prograde compositional zonation of garnet in whiteschist of the HP-UHPM Kokchetav massif, Kazakhstan: a record of progressive UHP metamorphism. Lithos, 52, 215-233.

Payne, M.C., Teter, M.P., Allan, D.C., Arias, T.A., and Joannopoulos, J.D. (1992) Iterative minimization techniques for $a b$ initio total-energy calculations: molecular dynamics and conjugate gradients. Reviews of Modern Physics, 64, 1045-1097.

Prokopenko, V.B., Dubrovinsky, L.S., Dmitriev, V., and Weber, H.P. (2001) In situ characterization of phase transitions in cristobalite under high-pressure by Raman spectroscopy and X-ray diffraction. Journal of Alloys and Compounds, 327, 87-95.

Rekhi, S., Dubrovinsky, L.S., and Saxena, S. (1999) Temperature-induced ruby fluorescence shifts up to a pressure of $15 \mathrm{GPa}$ in an externally heated diamond anvil cell. High Temperatures-High Pressures, 31, 299-305.

Ruiz-Cruz, M.D., and Sanz de Galdeano, C. (2012) Diamond and coesite in ultrahigh-pressure-ultrahigh-temperature granulites from Ceuta, Northern Rif, northwest Africa. Mineralogical Magazine, 76, 683-705.

Sharma, S., Mammone, J., and Nicol, M. (1981). Raman investigation of ring configurations in vitreous silica. Nature, 292, 140-141.

Smyth, J., and Hatton, C. (1977) Coesite-sanidine grospydite from Roberts-Victor kimberlite. Earth and Planetary Science Letters, 34, 284-290.

Sobolev, N.V., Fursenko, B.A., Goryainov, S.V., Shu, J.F., Hemley, R.J., Mao, H.K., and Boyd, F.R. (2000) Fossilized high pressure from the Earth's deep interior: The coesite-in-diamond barometer. Proceedings of the National Academy of Sciences, 97, 11875-11879.

Teter, D.M., and Hemley, R.J. (1998) High pressure polymorphism in silica. Physical Review Letters, 80, 2145-2148.

Veithen, M., Gonze, X., and Ghosez, Ph. (2005) Non-linear optical susceptibilities, Raman efficiencies and electrooptic tensors from first-principles density functional perturbation theory. Physical Review B, 71, 125107.

Wentzcovitch, R.M., Da Silva, C., Chelikowsky, J.R., and Binggeli, N. (1998) A new phase and pressure induced amorphization in silica. Physical Review Letters, 80, 2149-2152.

Wyckoff, R.W.G. (1963) Crystal Structures 1, 2nd ed., p. 7-83. Interscience Publishers, New York

Xiao, W., Chen, M., and Xie, X. (2011) Shock-produced coesite in the Xiuyan Crater, China. Meteoritics \& Planetary Science, 46, A256-A256.

Yagi, T., and Yamakata, M. (2000). Effect of hydristaticity on the phase transformations of cristobalite. In H. Aoki, Y. Syono, and R.J. Hemley, Eds., Physics Meets Mineralogy: Condensed Matter Physics in the Geosciences, Chapter 4.4, p. 242-256. Cambridge University Press, U.K.

MANUSCRIPT RECEIVED MAY 4, 2013

MANUSCRIPT ACCEPTED DECEMBER 7, 2013

MANUSCRIPT HANDLED BY SERgIo SPEZIALE 